\title{
Editorial
}

\section{Sensors for Robotics}

\author{
Aiguo Song, ${ }^{1}$ Guangming Song, ${ }^{1}$ Daniela Constantinescu, ${ }^{2}$ Lei Wang, ${ }^{3}$ and Quanjun Song ${ }^{4}$ \\ ${ }^{1}$ Robot Sensor and Control Lab, School of Instrument Science and Engineering, Southeast University, Nanjing 210096, China \\ ${ }^{2}$ Department of Mechanical Engineering, University of Victoria, P.O. Box 3055 STN CSC, Victoria, \\ British Columbia, Canada V8W 2 Y2 \\ ${ }^{3}$ School of Computer Science and Software Engineering, University of Wollongong, Wollongong, NSW 2522, Australia \\ ${ }^{4}$ Institute of Intelligent Machine, Chinese Academy of Sciences, Hefei 230031, China
}

Correspondence should be addressed to Aiguo Song; a.g.song@seu.edu.cn

Received 8 October 2013; Accepted 8 October 2013

Copyright ( $) 2013$ Aiguo Song et al. This is an open access article distributed under the Creative Commons Attribution License, which permits unrestricted use, distribution, and reproduction in any medium, provided the original work is properly cited.

Robots are currently widely applied in lots of areas such as industry, agriculture, medicine, transportation, social service, military, space exploration, and undersea exploiting. The robot sensor, as a key component of the robot, has been paid increasing attention by robot researchers. Furthermore, sensor based human-robot interaction is currently the frontier of robot research. Robot sensing technology has been an evolving research field involving a strong interdisciplinary effort from researchers in different fields, namely, electronics, mechanics, material science, measurement and control, signal processing, bioengineering, and so forth. During the last decade, much effort has been done to develop robot sensors for robot control, robot perception, robot navigation, human-robot interaction, teleoperational robot, and so forth. In spite of the large and increasing interest and promising applications, robot sensor design is a significant challenge, which is involved in not only chemical sensors, biosensors, optical sensors, and physical sensors but also signal processing, data fusion, and pattern recognition. For instance, remarkable examples of tactile sensors and systems have been proposed; however, their capability to address specific applications and their extension to other fields such as medical instrumentation, prosthetic devices, and augmented reality are questionable.

This special issue aims at exhibiting the latest research achievement, findings, and ideas in robot sensors. The special issue consists of 10 selected papers summarizing the most recent developments in the field of sensors for robotics. The contents of the special issue cover the main aspects of robot sensing technology such as robot vision, force sensor and tactile sensor, signal procession of robot sensor, robot sensing and environment modeling, and robot sensor applications.

Robot vision is a fundamental sensation of robot. The paper by Z. Zainal et al. presents a method of extracting information in estimating heading angle of vision system. Integration of grey-level cooccurrence matrix (GLCM) in area of interest selection is carried out to choose a suitable region that is feasible for optical flow generation. From the generated optical flow, heading angle is estimated and enhanced via moving median filter. They demonstrate that GLCM can improve the estimation result of the heading angle of vision system significantly. The paper by A. ChávezAragón et al. presents an approach for the automatic detection and fast 3D profiling of lateral body panels of vehicles. They introduce a method to integrate raw streams from depth sensors in the task of 3D profiling and reconstruction and a methodology for the extrinsic calibration of a network of Kinect sensors. This sensing framework is intended for rapidly providing a robot with enough spatial information to interact with automobile panels using various tools. The approach has been applied in the intelligent transportation industry, automated vehicle inspection, automotive production lines, and scan alignment and interpretation.

Force and tactile sensors are absolutely necessary elements for robot when interacting with environment, which call for large range and high accuracy amplifier. The paper by J. Liu et al. developed novel A/D conversion circuits for array tactile sensor by using nonlinear tent map phenomenon, which is characterized by sensitivity to small signal and nonlinear amplifying function. The tent map based A/D 
conversion circuit is very suited for multipath signal parallel sampling and A/D converting of large array tactile sensor. The paper by $\mathrm{H}$. Wang et al. describes a new sine fitting algorithm that is able to fit a multiharmonic acquired periodic signal. By means of a "sinusoidal wave" whose amplitude and phase are both transient, the "triangular wave" can be reconstructed on the basis of the Hilbert-Huang transform. This method can be used to test the effective number of bits of A/D converter, avoiding the trouble of selecting initial value of the parameters and working out the nonlinear equations. The paper by J. Ma et al. proposes a dynamic compensation method for improving the dynamic characteristic of two-axis force sensors. The operation frequency of the force sensor system is expanded by a digital filter with backward difference network. Compared to experiment data without compensation, the response time of the dynamic compensated data is reduced by $30 \% \sim 40 \%$.

Robots rely on sensors to provide them with information about their surroundings. However, high-quality sensors can be extremely expensive and cost prohibitive. Thus, many robotic systems have to use many lower-quality sensors. The paper by N. K. Malakar et al. presents an idea on how modeling a sensor can improve its efficacy when employed within a Bayesian inferential framework. They employ an autonomous robot arm as a test bed, which uses an inexpensive LEGO light sensor to estimate the position and radius of a white circle on a black field. They demonstrate that by incorporating an accurate model of the light sensor spatial sensitivity function (SSF) into the likelihood function of a Bayesian inference engine, an autonomous system can make improved inferences about its surroundings.

Robot sensing and environment modeling are always a key issue for robot navigation and autonomous behaviors. The paper by Y. Omura et al. presents a simple method to detect step height, slope angle, and trench width using four infrared-light-source PSD range sensors. In order to reduce the error of upward slope angle, they also propose a method to improve range-voltage performance of a range sensor. The experiments show that the step height can be measured with high accuracy by using this method. The paper by Malakar et al. proposes a relatively fast learning system in which a robot can autonomously construct a Q-space that considers the importance of the sensors. The paper defines the measure of importance of a sensor for a task, which is the correlation between the value of each sensor and reward in reinforcement learning. Consequently, the state space is reduced based on the importance of the sensors, thus allowing the robot to learn correspondences efficiently.

In this special issue, readers can also find some papers about the application of robot sensors in some important fields. The paper by T. Lehmann et al. developed a virtual sensor for minimally invasive surgery, which is able to precisely sense the needle tip deflection during needle insertion procedures using a 4 DOF force sensor installed on the needle base. The paper by $\mathrm{H}$. Nakamoto et al. presents an active touch method for robotic hands in order to improve dexterity performance of multifinger robotic hands. They define four requirements of a tactile sensor for active touch and design a distributed tactile sensor model, which is able to measure a distribution of compressive deformation. They also suggest a measurement process with the sensor model and a synthesis method of distributed deformations. The experiments of a five-finger robotic hand with tactile sensors that traces on the surface of cylindrical objects and evaluates the diameters show that the robotic hand can obtain more information about the diameters by tracing the finger.

\section{Acknowledgments}

We would like to thank all the authors for their valuable contributions and also the reviewers for their critical help necessary to achieve a high level of papers' quality and make thus possible the completion of this special issue. Also, we would like to thank all members of the Editorial Board for approving this special issue.

\author{
Aiguo Song \\ Guangming Song \\ Daniela Constantinescu \\ Lei Wang \\ Quanjun Song
}



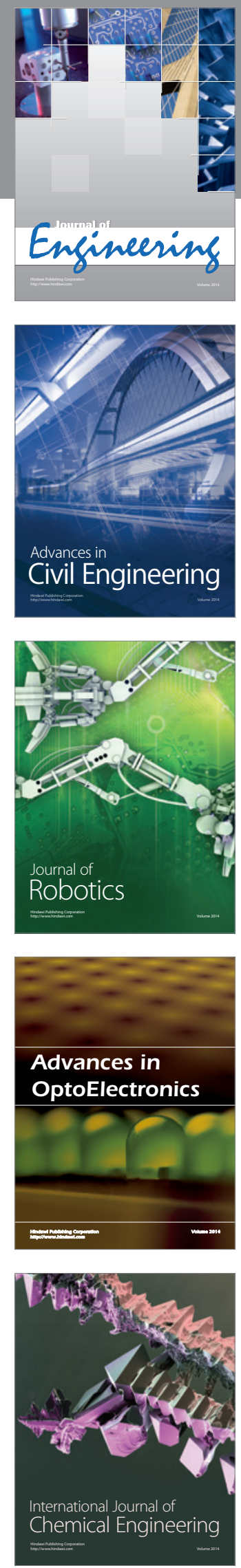

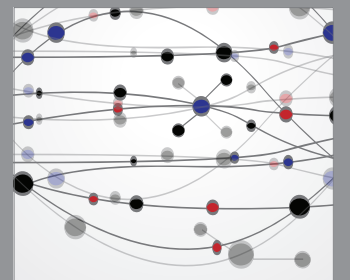

The Scientific World Journal
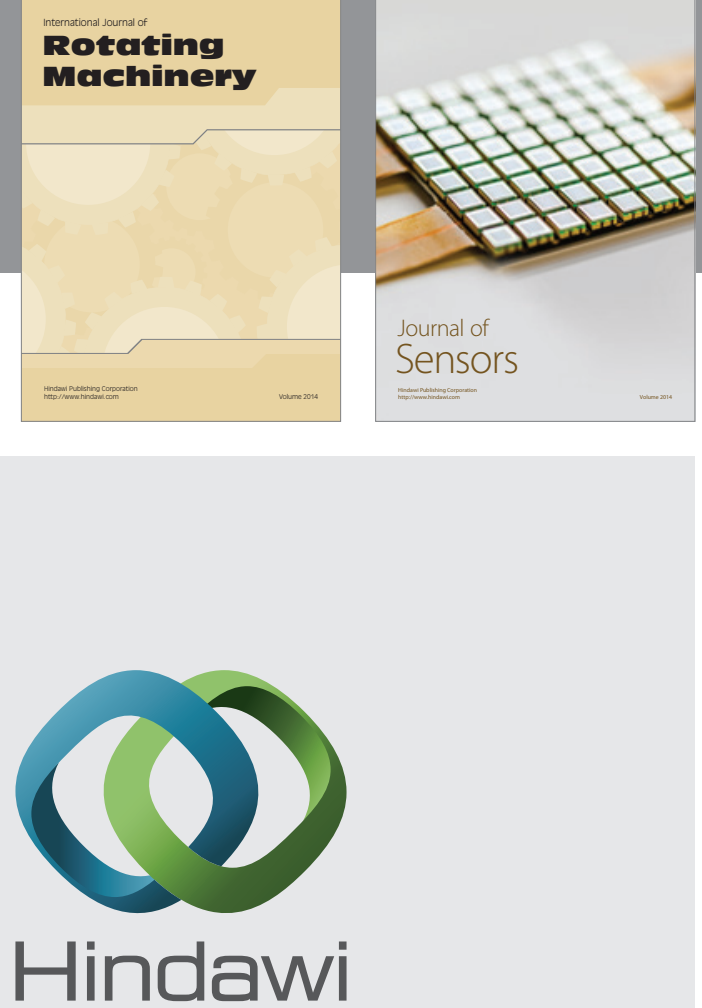

Submit your manuscripts at http://www.hindawi.com
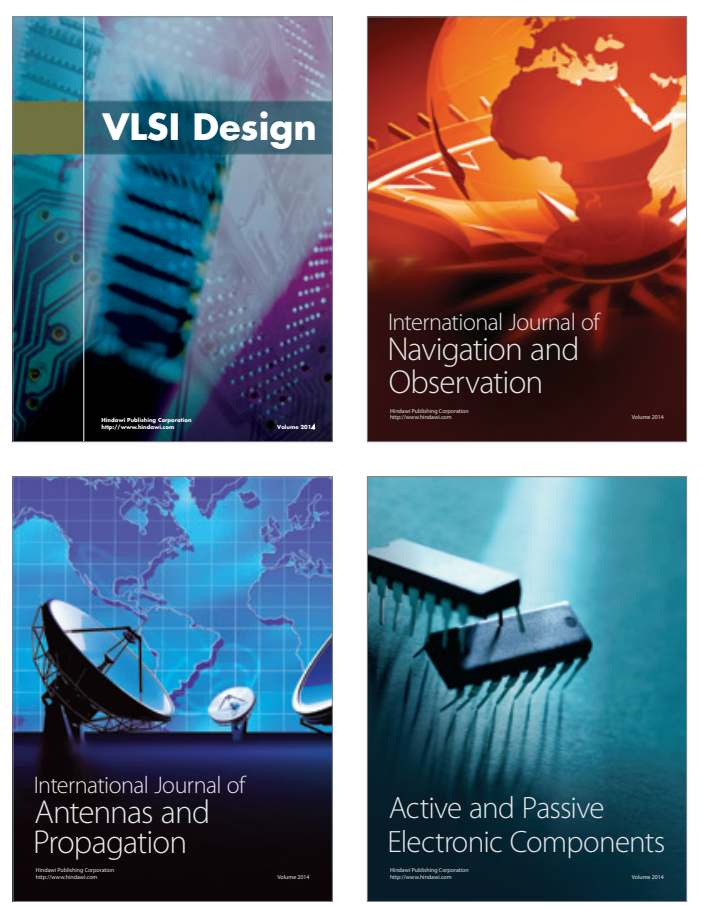
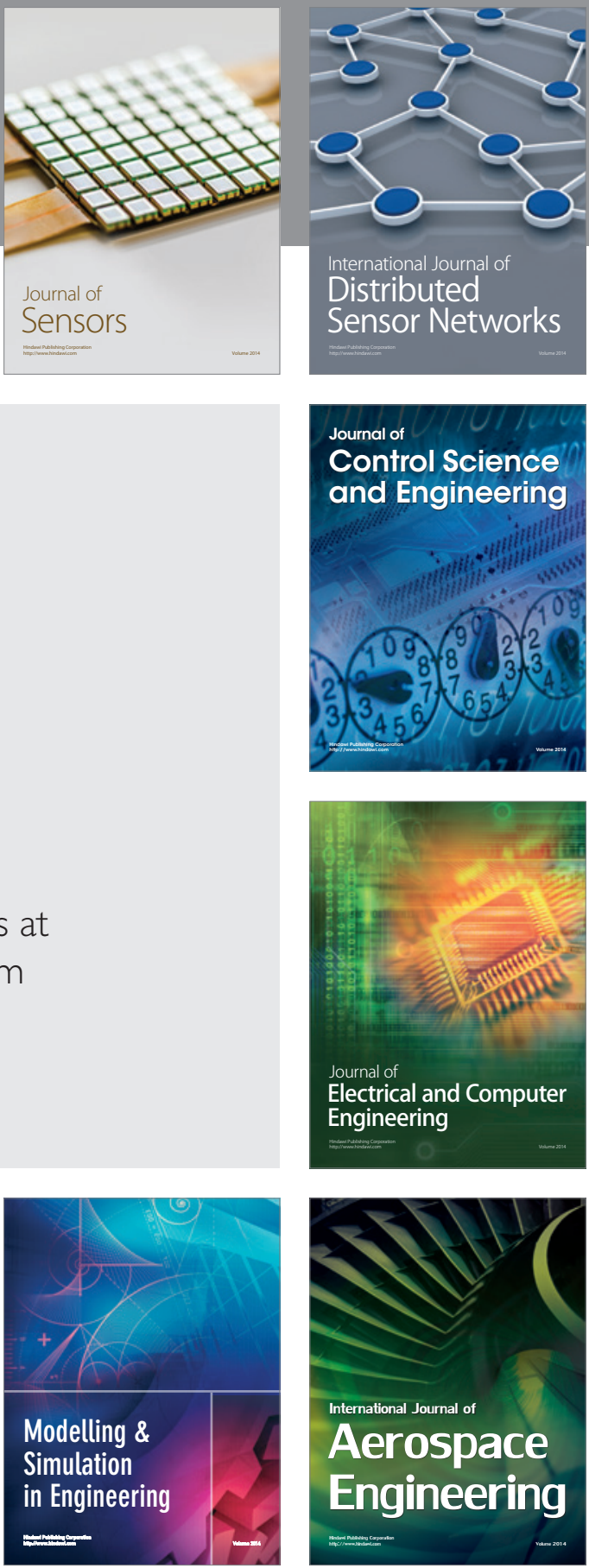

Journal of

Control Science

and Engineering
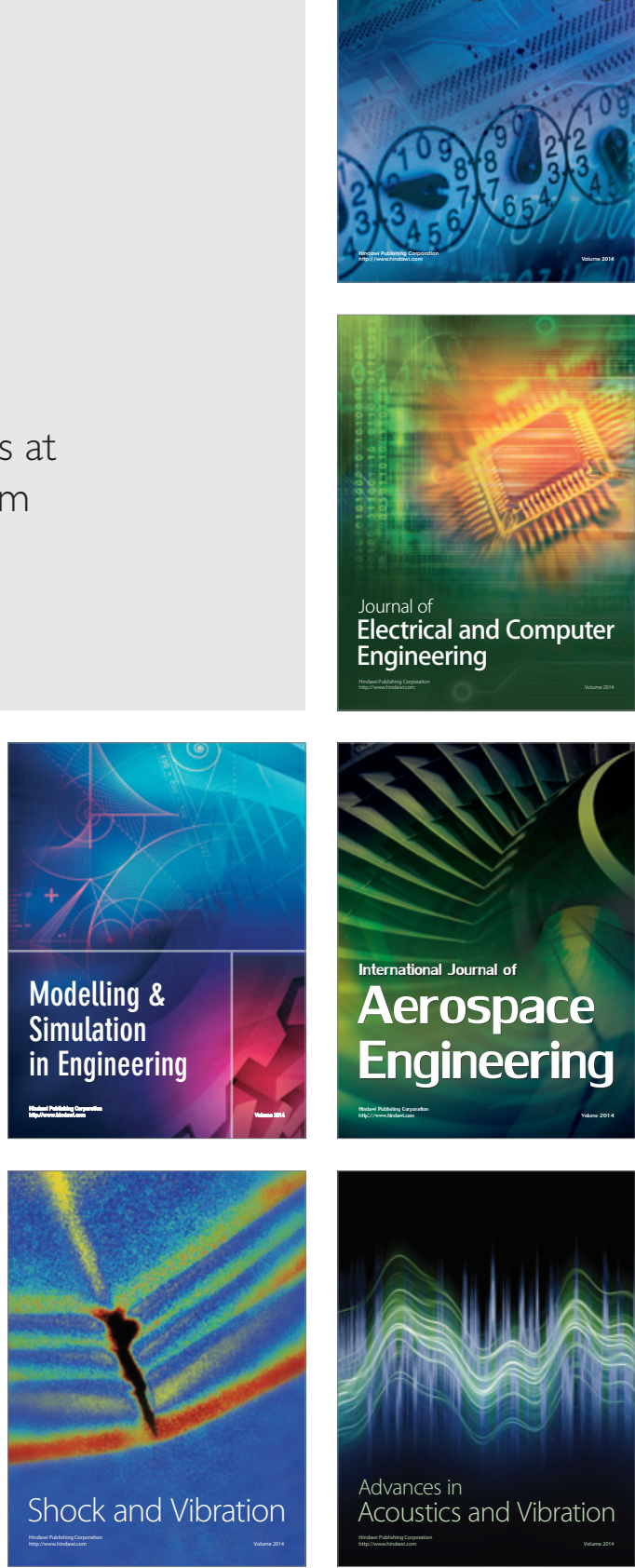